\title{
Effect of Temperature on the Twisting Moment of Nylon Yarn
}

\author{
By Saburo Manago and Kyuichiro Tanaka, Members, TMSJ
}

\author{
Faculty of Engineering, Kanazawa University
}

\begin{abstract}
This article discusses the effect of temperature on the twisting moment of twisted nylon and some other twisted yarns.

If heated twisted nylon, acetate and Terylene yarns are subjected to cooling at a given temperature in the one-time heating process, the twisting moment decreases with the decrease in temperature. In the process of re-heating following cooling, the moments of these yarns increase-until the temperature at which cooling was started is reached-along the moment-temperature curves which are identical with the curves representing the cooling effect. Above that temperature the moments change along the curves showing the effect of one-time heating.

On the other hand, the effect of temperature on twisted viscose rayon yarn is represented by a single moment-temperature curve.

These fundamental properties in the heat-setting of twist are discussed in this article.
\end{abstract}

\section{Introduction}

It is well-known that the twisting moment of nylon yarn is relaxed and twisted form is set by heat treatment. Heat treatment of twisted nylon yarn is important for the practical use of nylon yarn. There is little published information on the relation between the temperature of heat-treatment and the effects of setting on the twist. To use twisted nylon yarn for a special purpose, it seems necessary to know how twisting moment changes when previously heatset nylon yarn is re-heated.

Changes in the tension of the filament fibers of nylon yarn seem to be a basic problem calling for inquiry. Changes in tension are not equal in degree in all fillaments. Consequently, it may serve a useful purpose to inquire directly into the relation between the temperature of heat-treatment and the effects of setting on the twist. This article deals with changes in the twisting moment of nylon in dry state, when subjected to three processes: (1) heating, (2) heating and cooling, (3) heating, ccoling and re-heating. To compare the behaviors of nylon and other fibers, similar experiments have been made with viscose rayon, acetate and Terylene yarns.

\section{Apparatus Used and Methods of Experiments}

The yarns used in our experiments were the ordinary continuous filament yarns: nylon 110 denier, viscose rayon 120 denier, acetate 75 denier and Terylene 70 denier. The length of all specimens was initially $60 \mathrm{~mm}$ at zero twist. The apparatus is shown in Figure 1. The specimen was clamped to the metal rod attached to the lower end of the piano wire. The upper end of the piano wire was fixed immovably and the specimen laden at its lower end with a load of 6.9 grams. When the specimen is twisted at its lower end, the piano wire develops torsion and the small mirror attached to the metal rod rotates. The rota-

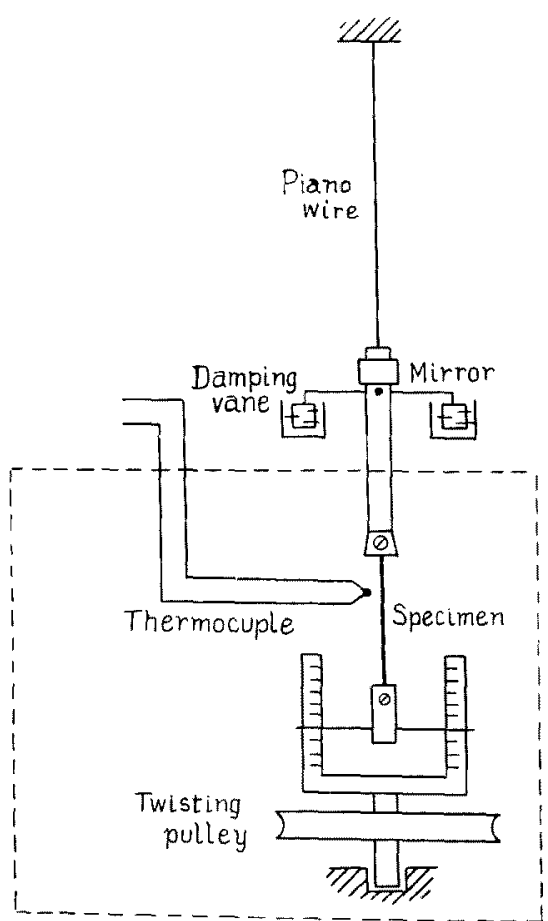

Fig. 1 Experimental apparatus 
tion of the mirror is observed by means of the telescope and scale, and the twisting moment of yarn is determined by applying the equation for the equilibrium of torsion wire. The twisting moment $M$ is given by the formula:

$$
\boldsymbol{M}=\frac{\pi a^{4} G}{2 l} \cdot \theta=\frac{\pi a^{4} G}{2 l} \cdot \frac{d}{2 D},
$$

$a$ is the radius of the piano wire, $l$ its length, $G$ its torsional rigidity, $d$ the deflection on the scale, and $D$ the distance between the mirror and the scale. The values used in our apparatus were:

$$
\begin{array}{ll}
a=1.525 \times 10^{-1} \mathrm{~mm}, & l=5.00 \times 10^{2} \mathrm{~mm} \\
G=8.77 \times 10^{3} \mathrm{~kg} \cdot \mathrm{mm}^{-2}, & D=1.012 \times 10^{3} \mathrm{~mm} .
\end{array}
$$

Some damping was added to the measuring system to prevent disturbance.

Since the actual temperature of the specimen was very difficult to measure, we regarded, as its temperature, the temperature represented by a copper-constantan thermo-couple placed $5 \mathrm{~mm}$ from the specimen. Changes in the length of the specimens by twisting and heattreatment is also observable by our apparatus. The specimens and some parts of the apparatus are placed in the heating oven. This is shown by the dashed line in Figure 1 . The twisting pulley is driven by the electric motor placed outside the oven.

A twist of 60 turns was given to the specimen at the room temperature, the rate of twisting being kept at about $3 / 4$ turn per second. After the twist madie its 60 th turn, the relaxation of the twisting moment of the yarn, kept at this constant twist, was observed for half an hour. Since relaxation slows down considerably after this period, heating of the specimens was begun forthwith and the observations described in the following paragraphs made.

\section{Results and Discussion}

\section{2-1. Relaxation of Twisting Moment}

Figure 2 shows the relaxation of the twisting moment for half an hour at $19 \pm 4^{\circ} \mathrm{C}, 75$ $\pm 5 \%$ R.H. With these variations in temperature and humidity, the initial twisting moment $M_{o}$ varies by several per cent. However, the ratio $M_{t} / M_{o}\left(M_{t}\right.$ is the moment at a given time) is affected little in such a small range of temperature and humidity - a fact which is analogous to the fact already observed on stress relaxation'1)

The results shown in Figure 2 are the average of 3 or 6 observations in the above-mentioned range of temperature and humidity. Viscose rayon and acetate yarns show greater relaxation than do nylon and Terylene yarns. There is a small difference in the relaxation properties of nylon between loads of $6.9 \mathrm{~g}$ and $16.9 \mathrm{~g}$. The decay of moment for each yarn is considerably linear to the logarithm of time. The curves for acetate and Terylene yarns are completely straight lines over the abserved range. Note, however, that acetate and Terylene yarns have lower deniers and that extensions of the filaments of these yarns are smaller.

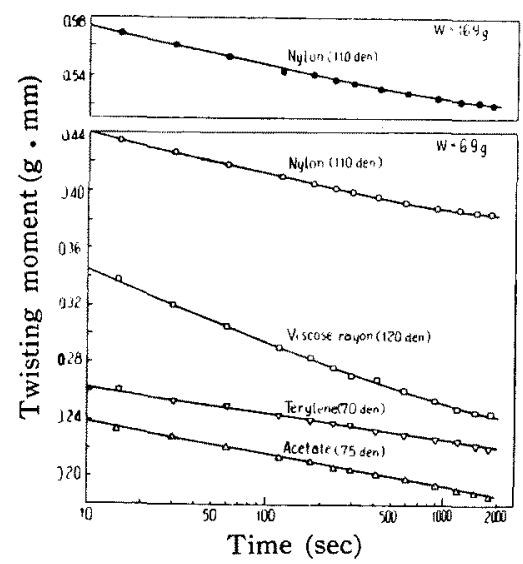

Fig. 2 Relaxation of twisting moment (twist: $10 t / \mathrm{cm}$ )

\section{2-2. Effect of Temperature on One-time Heating}

After relaxation for half an hour at the room temperature, a change in twisting moment by heating was observed. The results for nylon yarn were obtained at different rates of heating, $A$ and $B$ in Figure 3 . The results were the same for both rates of heating. $A$ is roughly a constant rate of heating of $7.8^{\circ} \mathrm{C} / \mathrm{min}$. Only this rate was used in the experiments described hereafter. Of course, the time required for heating to a given temperature varies with the room temperature. However, we have just noted that changes in twisting moment by heating at two different rates are the same in degree. From tnis fact we may conclude that the duration of heating does not affect the results of heating. On the other hand, our experiments show that nylon yarn relaxed for 18

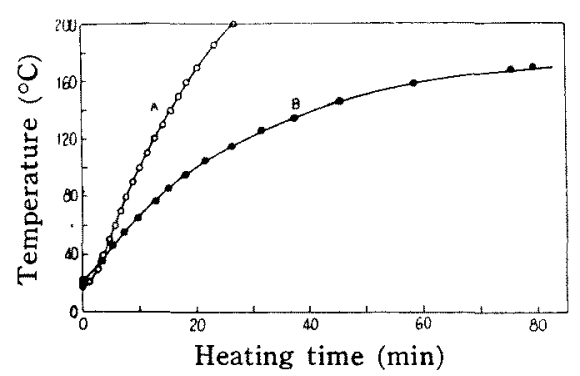

Fig. 3 Different rates of heating 
hours at the room temperature gives the same result as nylon yarn relaxed for half an hour. From these observations, we may also conclude that an observed change in twisting moment is due to a change in temperature and that a high temperature does not speed up relaxation.

The effects of one-time heating are shown in Figures 4 and 5. A specimen changes in length by heating, so that the number of twists per unit length does not remain at a constant value. Twisted nylon yarn shrinks by about $3.5 \%$ in length at $170^{\circ} \mathrm{C}$ from the length it has at the room temperature. Again, twisted nylon yarn with a load of $6.9 \mathrm{~g}$ ruptures at about $180^{\circ} \mathrm{C}$. Twisted "Terylene yarn shrinks by about $2.8 \%$ at $200^{\circ} \mathrm{C}$ and breaks at about $230^{\circ} \mathrm{C}$. Twisted viscose rayon yarm shows no change in length by heating. Twisted acetate yarn, by heating, develops a gradually increasing elongation which is as low as only a few per cent at $150^{\circ} \mathrm{C}$, compared with more than $10 \%$ at $170^{\circ} \mathrm{C}$.

Since the change in the length of yarns by heating is relatively small, as has just been shown, we may conclude that yarns remain at a constant number of twists during heat-treatment.

We know the influence of load from Figure 4. Under a load of $16.9 \mathrm{~g}$ there is a greater drop in moment at a high temperature. It seems, however, that the influence of a load of $6.9 \mathrm{~g}$ is very slight. The following observations and discussions are confined to a specimen with a load of $6.9 \mathrm{~g}$ imposed on it.

The curve for nylon is almost a straight

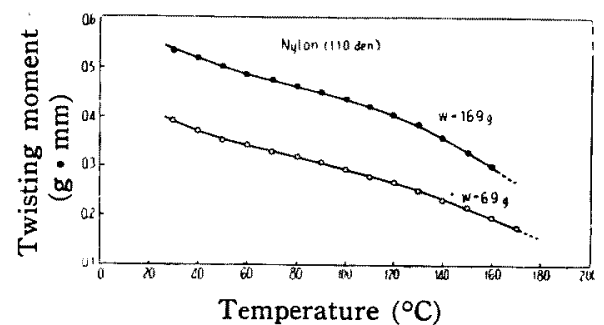

Fig. 4 Change in twisting moment by one-time heating (twist: $10 t / \mathrm{cm}$ )

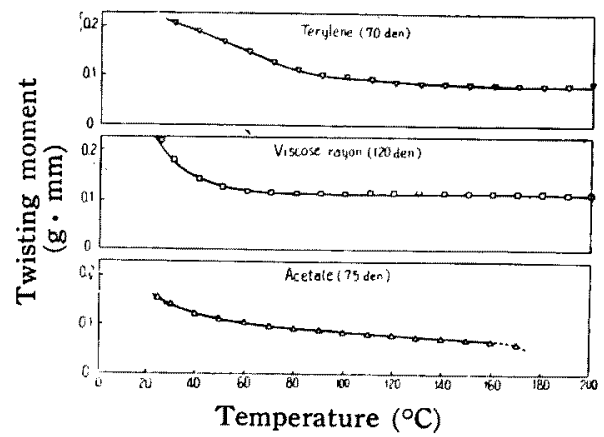

Fig. 5 Change in twisting moment by one-time heating (twist: $10 \mathrm{t} / \mathrm{cm}$, load: $6.9 \mathrm{~g}$ ) line. It seems that tnere is a slight drop in moment at the second-onder transition temperature $\left(49^{\circ} \mathrm{C}\right)$, but we have been unable to confirm this. E. Nagai and N. Sagane[2] have studied the effect of temperature on the tension of nylon filaments and report that tension shows a maximum value at the transition temperature. Although twisting moment results from the tension of yarn filaments, our experiments have shown no phenomena comparable with those observed by Nagai and Sagane. G. M. Bryant reports [3], however, that a cold-drawn nylon filament did not show a maximum tension at the transition temperature in a similar experiment made under water.

The curves for viscose rayon and acetate are similar in shape and show a considerable decrease in moment during heating up to about $60^{\circ} \mathrm{C}$. The twisting moments of visicose rayon and Terylene become nearly constant above $60^{\circ} \mathrm{C}$ and $120^{\circ} \mathrm{C}$, respectively. It is known that the second-order transition temperatures for viscose rayon, acetate and Terylene are $\left(60^{\circ} \mathrm{C}\right)$, $\left(60^{\circ} \mathrm{C}, 120^{\circ} \mathrm{C}\right)$ and $\left(69^{\circ} \mathrm{C}, 81^{\circ} \mathrm{C}\right)$, respectively. However, we can observe no clear relation between the transition temperature and a change in twisting moment.

\section{2-3. Effect of Rapid Cooling}

Immediately the heating process reaches a given temperature, open the door of the heating oven and switch off the electric circuit of the heating wire. Then, temperature drops rapidly. This is rapid cooling. The change in moment by rapid cooling was observed in our experiments. Nylon showed a sudden drop in moment by rapid cooling, but after a short time (about 1 minute), it showed a nearly constant value. The results of this observation are showen in Figure 6. The moment, by heating, decreases along the curve $A$, reaches value $\alpha$ and then, on rapid cooling at temperature $T$, drops to value $s$ on the curve $S$.

Consequently, the curve $S$ in Figure 6 shows a relation between the effect of heatsetting on twist in dry state and temperature. In the experiment about the effect of rapid cooling, the higher the temperature at which cooling was started, the higher the initial rate of cooling. For instance, cooling started at $160^{\circ} \mathrm{C}$ results in a temperature drop of about $50^{\circ} \mathrm{C}$ in 25 seconds. Acetate and Terylene show

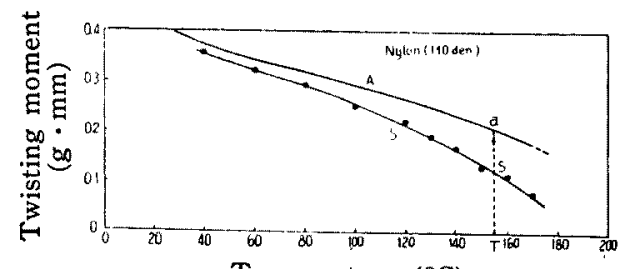

Temperature $\left({ }^{\circ} \mathrm{C}\right)$

Fig. 6 Effect of rapid cooling on twisting moment (twist: $10 \mathrm{t} / \mathrm{cm}$, load: $6.9 \mathrm{~g}$ ) 
behaviors similar to nylon's. Viscose rayon shows no drop in twisting moment.

\section{2-4. Effect of Re-heating Following Rapid Cooling}

When a specimen which had moment $s$ in Figure 6 , was heated at the room temperature which was raised gradually, a change in moment was observed. The results for nylon are shown in Figure 7 , wherein curves $B_{1}$ and $B_{3}$ correspond to specimens rapidly cooled at $100^{\circ} \mathrm{C}$ and $150^{\circ} \mathrm{C}$, respectively.

Twisting moment increases gradually by heating and reaches the orglnal moment $a$, at the starting temperature of rapid cooling, then decreases by further heating. This decrease, however, takes place along the one-time heating curve $A$.

We find, then, that previously heat-set yarn cannot take a higher moment than the moment at the starting temperature of cooling. In addition to these facts, we find that the change in moment is reversible on the curve $B$.

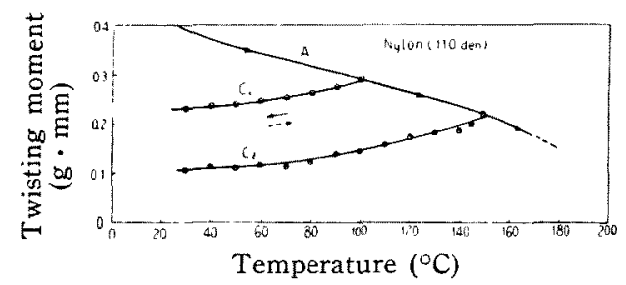

Fig. 7 Effect of re-heating following rapid cooling on twisting moment (twist: $10 \mathrm{t} / \mathrm{cm}$, load:6.9g)

\section{2-5. Effect of Slow Cooling}

As soon as the heating process reaches a given temperature, switch off only the electric circuit but keep the door of the heating oven closed. Then, temperature drops slowly. This is slow cooling. The curves $C_{1}$ and $C_{2}$ in Figure 8 are obtained for nylon cooled slowly from $100^{\circ} \mathrm{C}$ and $150^{\circ} \mathrm{C}$, respectively. In this experiment, it took from 80 to 100 minutes for cooling to $30^{\circ} \mathrm{C}$.

Note that the moment on the curve $C$ at the room temperature takes nearly the same value as for rapid cooling at each different starting temperature of cooling. The behaviors of

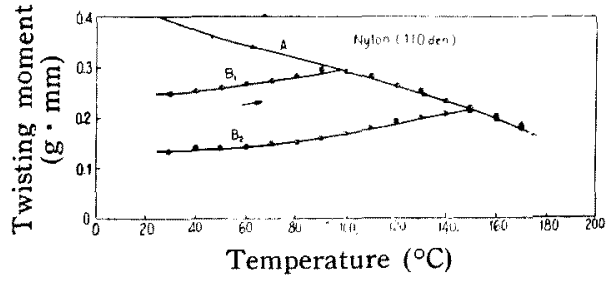

Fig. 8 Effect of slow cooling on twisting moment (twist: $10 \mathrm{t} / \mathrm{cm}$, load: $6.9 \mathrm{~g}$ ) acetate and Terylene, shown in Figure 9, are similar to nylon's. Viscose rayon, however, differs in behavior from other fibers, in that its change in moment takes place along the onetime heating curve, both in the process of slow cooling and in the process of re-heating following slow cooling.

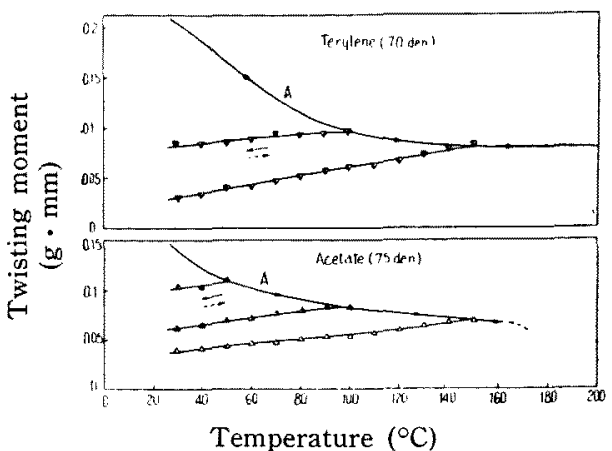

Fig. 9 Effect of slow cooling on twisting moment (twist: $10 \mathrm{t} / \mathrm{cm}$, load: $6.9 \mathrm{~g}$ )

\section{2-6. Effect of Re-heating Following Slow Cooling}

Re-heating is started immediately the temperature of the slow cooling process reaches the room temperature. The moments for nylon, Terylene and acetate, until the starting temperature of cooling is reached, change along the curves representing the effects of slow cooling. Above that temperature they change along the one-time heating curve. Therefore, there is a reversible change along the curve $C$ and a behavior similar to that observed in re-heating following rapid cooling.

It may be concluded from these facts that the curve $B$ in Figure 7 is identical with the curve $C$ in Figure 8 ; and that such a behavior is uninfluenced by the rate of cooling used in our experiments. Inis result corresponds to the fact that the rate of heating does not affect the results of the one-time heating. In the authors' opinion, the decrease in twisting moment by rapid cooling also takesplace along the curve $C$. S. Ueda and T. Kimura [4] have measured the strength, density and other properties of nylon heat-treated under various cooling conditions and found that the rate of cooling does not affect the results appreciably. They have also found that the chain molecule in a nylon fiber is ruptured by oxidation through heating in the air. However, we may conclude that this rupture of the chain molecule does not affect the twisting moment, since the change in moment by re-heating following cooling takes place along the one-time heating curve above the starting temprature of cooling.

The effect of temperature on viscose rayon is represented by a single reversible curve. This fact supports the following points of view: 
1. The temperature of a specimen is nearly the same as the temperature indicated by the thermo-couple thermometer.

2 . The influence of the moisture content of a specimen is negiigible.

3. Stress relaxation contributes but little to the observed change in the twisting moment. This last point is also supported by the fact that the moments for nylon, acetate and Terylene, above the starting temperature of cooling, change along the one-time heating curve $A$.

The tension of fiber can be devided into parts depending on internal energy $E$ and entropy $S$. Thus each contribution may be thought of separately in terms of the following thermo-dynamic relation:

$$
\begin{aligned}
f & =\left(\frac{\partial E}{\partial l}\right)_{T}-T\left(\frac{\partial S}{\partial l}\right)_{T} \\
& =\left(\frac{\partial E}{\partial l}\right)_{T}+T\left(\frac{\partial f}{\partial T}\right)_{T} .
\end{aligned}
$$

where $l$ is the length and $T$ the absolute temperature. Since the term depending on the change of entropy $T(\partial f / \partial \tau)$ can be measured by observing the temperature coefficient of tension in constant length, the term depending on the change of internal energy $(\partial E / \partial !)_{T}$ is also obtained from the above relation. G. M. Bryant and H. Wakeham, (3) (5) measured the temperature coefficient of the tension of nylon in water and applied the above relation to the tension-temperature curve which showed the reversible change. This reversible curve was obtained on a specimen which had been relaxed for a period of time at the highest temperature used in their experiments.

We may be allowed, by using the curves which show the reversible change of moment, to assess the relative importance of internal energy and entropy in contributing to the twisting moment. Since the temperature coefficient of the twisting moment has a positive sign for nylon, acetate and Terylene, it appears that the moment depending on entropy may be more important to these fibers. However, the moment for viscose rayon may be dominated by internal energy. The curve $C_{t}$ in Figure 8 is nearly parallel to the curve $C_{2}$. Consequently, in cases where the highest temperatures in the reversible ranges are at $100^{\circ} \mathrm{C}$ and $150^{\circ} \mathrm{C}$, respectively, the contribution of entropy to the moment will be, in both cases, nearly the same at a given temperature. It seems that twisted acetate yarn has also a property similar to nylon's.

E. Nagai and N. Sagane $\{2\}$ have observed that the tension of undrawn nylon increases by cooling. It seems, then, that it is impossible to give twist-setting on undrawn nylon.

\section{Conclusions}

1. On one-time heating the twisting moment of yarn decreases with an increase in temperature. However, the moment of twisted Terylene and twisted viscose rayon yarns remains constant beyond $120^{\circ} \mathrm{C}$ and $60^{\circ} \mathrm{C}$, respectively. There is no clear relation between a change in moment and the second-order transition temperature of fiber.

2. Twisted nylon, Terylene and acetate yarns, if subjected to cooling at any temperatures on the one-time heating process, decrease in twisting moment with the decrease in temperature. The higher the starting temperature of cooling, the larger drop in twisting moment. Thus the twist-setting of nylon, Terylene and acetate yarns is possible by heat-treatment in their dry state.

3. By re-heating following cooling, the moment of twisted nylon, acetate and terylene yarns, until the stariing temperature of cooling is reached, increase along the moment-temperature curves identical with those representing the effects of cooling. Above that temperature the moments change along the one-time heating curves.

4. Viscose rayon differs in behavior from other fibers, in that the change in moment by cooling and re-heating is represented by the curve identical with the one-time heating cruve. Thus viscose rayon shows no drop in twisting moment by cooling.

\section{Literature cited}

[1] S. Manago, K. Tanaka; J. Text. Maoh. Soc., Japanese edition, Vol. 10, No. 1, p. 46 (1957)

[2] E. Nagai, N. Sagane; J. Soc. Text. Celluloge Indust., Japan, Vol. 8, No. 9, p. 429 (1952)

[3] G. M. Bryant; Text. Res. J., Vol 23, No. 11, p. 788 (1953)

[4] S. Ueda, T. Kimura; Bull. Text. Res. Inst., Japan, No. 30, p. 1 (1954)

[5] G. M. Brylant, H. Wakeham; Text. Res. J., Vol. 25, No. 3, p. 224 (1955) 